\title{
Pediatric Traumatic Cataracts at a Tertiary Eye Center in Indonesia
}

\author{
Feti Karfiati Memed ${ }^{1,2 *}$, Desi Mariska², Maya Sari Wahyu ${ }^{1,2}$, Irawati Irfani1,2, \\ Primawita Oktarima ${ }^{1,2}$, Sesy Caesarya ${ }^{1,2}$ \\ ${ }^{1}$ Pediatric Ophthalmology and Strabismus Unit of National Eye Center Cicendo Eye Hospital, Bandung, Indonesia \\ ${ }^{2}$ Ophthalmology Department, Faculty of Medicine Universitas Padjadjaran, Bandung, Indonesia \\ Email: ^fkmemed@gmail.com
}

How to cite this paper: Memed, F.K., Mariska, D., Wahyu, M.S., Irfani, I., Oktarima, P. and Caesarya, S. (2020) Pediatric Traumatic Cataracts at a Tertiary Eye Center in Indonesia. Open Journal of Ophthalmology, 10, 332-340.

https://doi.org/10.4236/ojoph.2020.104036

Received: September 24, 2020

Accepted: November 9, 2020

Published: November 12, 2020

Copyright ( 2020 by author(s) and Scientific Research Publishing Inc. This work is licensed under the Creative Commons Attribution International License (CC BY 4.0).

http://creativecommons.org/licenses/by/4.0/

\begin{abstract}
Background: Traumatic cataract is the leading cause of significant monocular visual impairment in children. The cause of this type of cataract is preventable penetrating or blunt ocular injury. Lens extraction can improve the visual acuity but it also depends on the extent of the injury to other ocular structures. Objective: To describe the features of pediatric traumatic cataract presenting at a tertiary eye center in Indonesia. Methods: This study is a descriptive study and the data were collected retrospectively from the medical records of the patients who were diagnosed as pediatric traumatic cataract over the period of January $1^{\text {st }} 2019$ to December $31^{\text {st }} 2019$. Demographic data, trauma characteristics, clinical features, management, and pre- and post-operative Best Corrected Visual Acuity (BCVA) were reviewed retrospectively. Results: A total of 37 patients were diagnosed as pediatric traumatic cataract. Among these patients, $78.38 \%$ were boys, with the mean age of $9.14 \pm 3.77$ years old. Open globe injury was the mechanism of injury for $54.05 \%$ patients. Besides lens aspiration, additional procedures were membranectomy, anterior vitrectomy, primary posterior capsulotomy, and synechiolysis. Eighty-one percent patients had unilateral blindness preoperatively and $23.80 \%$ patients still had unilateral blindness on three months of follow-up. Conclusion: In pediatric patients, traumatic cataract occurred predominantly in boys while playing outside the house. The children who had ocular trauma still have the risk of blindness even after the surgery. Trauma prevention and avoidance by adult supervision when children engage in outdoor play activity are necessary.
\end{abstract}

\section{Keywords}

Traumatic Cataract, Pediatric, Visual Outcome 


\section{Introduction}

Ocular trauma is the leading cause of unilateral blindness in children [1]. About $20 \%-50 \%$ of ocular trauma occurs in the pediatric age group, and at least $90 \%$ of ocular trauma cases in children can be prevented [2] [3] [4] [5]. Cataracts in children result in more than one million blindness among Asian children [5]. A traumatic cataract is defined as permanent lens opacity due to blunt or penetrating trauma to the eye. Traumatic cataracts occur in $29 \%$ - 57\% of all cataract cases in children. They are the cause of visual impairment in children, especially in developing countries, and often cause more significant visual impairment compared to other types of cataract. This is because traumatic cataracts are usually accompanied by other ocular disorders such as corneal lacerations, iris injury, vitreous hemorrhage and retinal detachment [2] [3] [6] [7] [8].

The treatment of pediatric traumatic cataracts is challenging since these cataracts occur in the developing eyes, which is at risk of amblyopia. Traumatic cataracts can provide good visual acuity if treated with optimal surgery and timely visual rehabilitation. Postoperative visual acuity from previous studies varied, with final visual acuity $\geq 6 / 12$ as much as $50 \%-80 \%$ [2] [7] [9]. To our knowledge, there was no previous study which describes the characteristics of pediatric traumatic cataracts in Indonesia. This study aims to describe the overview of pediatric traumatic cataracts at the Indonesia National Eye Center Cicendo Eye Hospital, which is the highly rated referral eye hospital in Indonesia.

\section{Subjects and Methods}

This is a descriptive study through retrospective data collection from medical records of all patients diagnosed with pediatric traumatic cataracts at Cicendo Eye Hospital Bandung from January $1^{\text {st }}, 2019$, to December $31^{\text {st }}$, 2019. The study adhered to the tenets of the Declaration of Helsinki and was approved by the Institutional Review Board (IRB) Universitas Padjadjaran. Inclusion criteria included all patients clinically diagnosed as traumatic cataract and aged 18 years or less. Exclusion criteria were patients with a history of other ocular abnormalities before the trauma, a history of ocular surgery before ocular trauma, and an incomplete medical record.

Pediatric traumatic cataracts are defined as lens clouding found on ophthalmological examination with a history of penetrating or blunt trauma in the age group of 18 years or less. Types of trauma are divided into traffic accidents, falls, and trauma due to blunt objects and sharp objects. A traffic accident is a trauma caused by an accident while driving, whereas fall is caused by falling from a height. Trauma due to blunt objects is caused by a hard object with a non-pointed tip. Meanwhile, sharp objects are objects with thin edges, smooth and easy to cut.

Data were taken retrospectively, consisting of data on age, gender, etiology and type of trauma, clinical characteristics, type of treatments given, and visual acuity before and after the procedure. Visual acuity in children was conducted using a Snellen chart, Cardiff visual acuity card or other pre-verbal visual acuity 
examination according to the child's ability. The data obtained were then presented by tables using Microsoft Excel 2016. The etiology and laterality variables were analyzed and statistically tested using the IBM SPSS version 27 with the Saphiro Wilk test.

\section{Results}

A total of 37 pediatric traumatic cataract patients were included in this study. This accounted for $13.19 \%$ of all pediatric cataract patients in 2019 at this hospital. Patient demographic data are presented in Table $1.78 .38 \%$ of all pediatric traumatic cataract patients were male. The average age of the patients was $9.14 \pm$ 3.77 years with an age range of 3 to 16 years. $48.65 \%$ of patients belong to the 6 to 10 -year age group. As many as $54.05 \%$ of cases were caused by open globe injuries (OGI) and $45.95 \%$ due to closed globe injuries ( $p=0.63$ ). In terms of locations, trauma occurred outside the building accounted for $72.93 \%$ of patients. Also, blunt objects were the cause of trauma in $78.38 \%$ of cases. In this study, all traumatic cataracts occurred unilaterally with $54.05 \%$ occurring in the right eye and $45.95 \%$ in the left eye $(\mathrm{p}=0.63)$.

Table 1. Demographic data and trauma characteristics.

\begin{tabular}{|c|c|}
\hline Characteristic & Number (\%) \\
\hline \multicolumn{2}{|l|}{ Gender } \\
\hline Male & $29(78.38)$ \\
\hline Female & $8(21.62)$ \\
\hline \multicolumn{2}{|l|}{ Age } \\
\hline 0 - 5 years old & $5(13.51)$ \\
\hline 6 - 10 years old & $18(48.65)$ \\
\hline $11-15$ years old & $11(29.73)$ \\
\hline $16 \leq 18$ years old & $3(8.11)$ \\
\hline Mean & $9.14 \pm 3.77$ \\
\hline Range & $3-16$ years \\
\hline \multicolumn{2}{|l|}{ Etiology type } \\
\hline Open globe injury & $20(54.05)$ \\
\hline Closed globe injury & $17(45.95)$ \\
\hline \multicolumn{2}{|l|}{ Trauma type } \\
\hline Traffic accident & 0 \\
\hline Fall & 0 \\
\hline Blunt object & $29(78.38)$ \\
\hline Sharp object & $8(21.62)$ \\
\hline \multicolumn{2}{|l|}{ Location } \\
\hline Inside building & $10(27.03)$ \\
\hline Outside building & $27(72.97)$ \\
\hline \multicolumn{2}{|l|}{ Laterality } \\
\hline Right eye & $20(54.05)$ \\
\hline Left eye & $17(45.95)$ \\
\hline
\end{tabular}


Table 2 shows the characteristics of traumatic cataracts in this study. A total of $37.84 \%$ had anterior capsule rupture. In $97.30 \%$ of patients, lens aspiration and irrigation were performed with the anterior approach. Meanwhile, in $94.60 \%$ of patients, IOL implantation was conducted.

Table 3 shows the preoperative and postoperative visual acuity. At the preoperative stage, as many as $81.08 \%$ of patients had visual acuity $<3 / 60$. Afterwards, at three-month-postoperative follow-up, it was found that $47.62 \%$ of 21 patients who came for follow-up had visual acuity $\geq 6 / 12$, while five patients remained to have visual acuity $<3 / 60$.

Table 2. Characteristics of pediatric traumatic cataracts.

\begin{tabular}{cc}
\hline Characteristic & Number (\%) \\
\hline Cataract Morphology & $7(18.92)$ \\
Membranous & $4(10.81)$ \\
Posterior subcapsular cataract & $14(37.84)$ \\
Anterior capsule rupture & $4(10.81)$ \\
Posterior capsule rupture & $9(24.32)$ \\
Total cataract & \\
Surgery (Lens Extraction) & $36(97.30)$ \\
Anterior approach (corneal) & $1(2.70)$ \\
Pars plana lensectomy & \\
Additional Surgery & $13(35.14)$ \\
Membranectomy & $10(27.03)$ \\
Anterior vitrectomy & $7(18.92)$ \\
Primary posterior capsulotomy & $13(35.14)$ \\
Synechiolysis & \\
IOL implantation & $35(94.60)$ \\
Pseudophakia & $21(60)$ \\
In the bag & $14(40)$ \\
Ciliary suclus & $2(5.40)$ \\
Aphakia & \\
\hline
\end{tabular}

IOL: Intraocular Lens.

Table 3. Pre-operative and post-operative best corrected visual acuity.

\begin{tabular}{ccccc}
\hline & Pre-operative & \multicolumn{3}{c}{ Post-operative } \\
\cline { 2 - 5 } BCVA & $\begin{array}{c}\text { Number (\%) } \\
(\mathrm{n}=37 \text { patients })\end{array}$ & $\begin{array}{c}\text { 1 week } \\
\text { Number (\%) }\end{array}$ & $\begin{array}{c}1 \text { month } \\
\text { Number }(\%)\end{array}$ & $\begin{array}{c}\text { 3 months } \\
\text { Number }(\%)\end{array}$ \\
$(\mathrm{n}=\mathbf{3}$ patients $)$ & $\mathbf{3 4}$ patients $)$ & patients) \\
\hline$<3 / 60$ & $30(81.08)$ & $9(24.32)$ & $9(26.47)$ & $5(23.80)$ \\
$3 / 60-<6 / 60$ & $1(2.70)$ & $1(2.70)$ & 0 & 0 \\
$6 / 60-<6 / 18$ & $5(13.52)$ & $5(13.52)$ & $4(11.76)$ & $3(14.29)$ \\
$6 / 18-<6 / 12$ & 0 & $11(29.73)$ & $5(14.71)$ & $3(14.29)$ \\
$\geq 6 / 12$ & $1(2.70)$ & $11(29.73)$ & $16(47.06)$ & $10(47.62)$ \\
\hline
\end{tabular}




\section{Discussion}

Traumatic cataracts can result from penetrating or blunt force trauma to the lens capsule and/or lens. The cataracts can occur days to years after trauma. Patients with significant traumatic cataracts can show good outcomes if treated appropriately [2] [10].

This study showed that the majority of traumatic cataract patients were boys at $78.38 \%$. This is in line with previous studies carried out by Gogate et al., Adlina et al., and Shah et al. which reported that $61.7 \%, 82.76 \%$, and $70.9 \%$ of patients were boys in their respective studies. This is as a result of boys spending more time in outdoor activities and playing more violent games than girls [6] [7] [11] [12].

Traumatic cataract patients in this study were mostly in the age group of 6 10 years with an age range of $3-16$ years. Adlina et al. also reported that the age range for the occurrence of traumatic cataracts was 3 - 17 years [7]. The study by $\mathrm{Xu}$ et al. reported that $58.97 \%$ of patients were in the 4 to 9 -year age group [4]. The high number of cases at pre-school age is due to children's curiosity to explore the objects around them without adult supervision. Meanwhile, at school age, many children are exposed to activities or games outside the home, which make them more vulnerable to trauma [6] [7] [12] [13] [14].

The causes of traumatic cataracts can be divided into open globe injury (OGI) and closed globe injury (CGI), depending on the presence or absence of perforation on the outside of the eyeball [6] [11] [15]. This study reported that the etiology by OGI and CGI did not differ significantly. A study by Shah et al. showed that $81.07 \%$ of patients were included in OGI [11]. Adlina et al. reported that $62.07 \%$ of patients had penetrating ocular injuries [7]. This is different from the study of Gogate et al., which stated that only $21.9 \%$ of cases were caused by OGI [6].

Several previous studies have shown mixed results when comparing the prognosis of traumatic cataracts due to OGI and CGI. Shah et al. stated that there was no difference in visual prognosis between OGI and CGI [16] [17]. Khokhar et al. claimed that there was no difference in visual acuity and post-surgical complications between OGI and CGI; however, CGI had a less residual refractive error [2]. Kinori et al. reported that traumatic cataracts due to blunt trauma objects have a better prognosis [3]. Meanwhile, our study has not performed a prognosis comparison between cases caused by OGI and CGI.

In this study, types of object responsible for the ocular trauma varied. Sharp objects included needles, fishing hooks, nails, screwdrivers, and knives. Most of the trauma in this study was caused by blunt objects consisting of toys, firecrackers, fruit, wood, and punches. Previous studies concluded that the most causative objects were wooden sticks and sharp objects [6] [8] [11].

This study showed that $72.97 \%$ of trauma events occurred outside the building. This is consistent with a study by Adhikari et al., which reported that $61.7 \%$ of incidents happened outside the building and occurred while playing [13]. Ad- 
lina et al. stated that trauma often occurs outside the building, especially in children over seven years [7].

In this study, all patients experienced traumatic cataracts unilaterally, which is consistent with previous studies showing the unilaterality of traumatic cataracts. Our study shows that the number of patients with traumatic cataracts in the right and left eyes is almost the same. This is similar to the study of Gogate et al., which showed no difference in the incidence rate between the right and left eyes [6]. However, it contradicts the study by Adlina et al. and Xu et al. which reported that $62.07 \%$ and $56.41 \%$ cases occurred in the right eye, whereas Khokhar et al. reported that $58 \%$ cases occurred in the left eye [2] [4] [7].

The morphology of traumatic cataracts can vary to include total cataract, membranous, rosette, and capsule rupture accompanied by lens cortex material in the front chamber of the eye [17]. A total of $37.84 \%$ of patients in this study had anterior capsule rupture. The study by Reddy et al. showed that $56 \%$ of patients had damages to the anterior capsule [9]. Cataract morphology in the study of Khokhar et al. showed that $58.1 \%$ were partially absorbed cataracts in the OGI group and $42 \%$ were total cataracts in the CGI group [2].

The surgical procedure of traumatic cataracts consists of lens extraction with or without posterior capsule support. The most used procedure in traumatic cataracts is by irrigation and lens aspiration [6] [16]. This study showed that 36 patients underwent lens aspiration and irrigation, and only one patient underwent pars plana lensectomy since it was accompanied by subluxation of the lens to the posterior eye segment. Shah et al. stated that surgery from the anterior approach gives better results than that from the posterior approach [16]. In our study, patients who underwent pars plana lensectomy were included in the final BCVA group at 1 -month follow-up $<3 / 60$ because they were accompanied by retinal detachment.

Gogate et al. reported that $22 \%$ of patients requiring more than one surgical intervention. Following this study, all traumatic cataract patients who were accompanied by corneal lacerations were treated with two-stage surgery. When cataract extraction is performed in conjunction with the closure of the ocular wall laceration, some experts recommend that intraocular lens (IOL) implantation be performed at the same time. To put it into consideration, IOL can help the survival of bacteria that enter the intraocular area during trauma [6] [10].

In this study, $94.60 \%$ of patients had simultaneous IOL implantation with cataract extraction, and most of these could be done in a capsular bag. This is in line with the study of Khokhar et al., which claimed that $86 \%$ of patients could undergo IOL implantation [2]. Previous studies have suggested that IOL implantation is associated with better visual prognosis. Patients who underwent primary IOL insertion were said to be less likely to have strabismus or binocular visual impairment [18]. Biometric examination, implantation of the IOL in the eye of a child who is still developing, and the possibility of amblyopia are significant challenges in IOL implantation in children traumatic cataracts [4] [6] [9] 


\section{[10] [15].}

Sen et al. suggested that there was no difference in visual acuity and refractive status in patients who underwent primary or secondary IOL implantation [18]. However, when placing the IOL in traumatic cataract patients, the support of the lens capsule to allow for IOL insertion in the capsular bag or the ciliary sulcus should be considered. If the posterior capsule support is inadequate, then the technique of choice in IOL placement includes IOL in the anterior chamber, iris fixation or sclera fixation [9] [17] [18]. Sixty per cent of the patients in this study had IOL in the capsule bag and $34.29 \%$ in the ciliary sulcus. Two patients were still in a state of aphakia, consisting of 1 one patient of post-pars plana lensectomy and one patient with IOL instability at the intraoperative time. The visual acuity of pediatric traumatic cataract patients varies from various studies [7] [11] [15] [16] [17]. This study showed that $47.62 \%$ of patients had visual acuity $\geq$ $6 / 12$ at three-month follow-up. This may not represent the actual situation because the decreased number of patients who underwent examination after three months of follow up was only 21 out of 37 patients. Many patients came from the location that was far from this national eye center and this is responsible for the large number of drop-off of patients by month three.

Gradin et al. reported that $64.7 \%$ of patients had better than 20/60 visual acuity after traumatic cataract surgery [19]. Meanwhile, Gogate et al. stated that $46.3 \%$ of patients had visual acuity more than $20 / 60$ postoperatively [6]. Aldakaf et al. concluded that initial visual acuity and the mechanism of trauma were predictors of visual outcome [20]. Eyes traumatized by sharp objects have a more unsatisfactory visual outcome. This also occurs in eyes that require more than one surgery [6] [19].

Adlina et al.'s study revealed that only $34.48 \%$ of patients achieved Best Corrected Visual Acuity (BCVA) $\geq 6 / 12$ [7]. A similar study by Shah et al. also reported that about $35 \%$ of patients had final BCVA $\geq 6 / 12$ within six weeks [11]. The causes of poor final visual acuity in this study included corneal opacification and amblyopia. This study resulted in five patients with final visual acuity $<3 / 60$ at three-month follow-up. The comorbid ocular conditions seen in these patients included two deprivation amblyopia patients, one patient with fibrotic tissue in front of the optic disc, one patient with corneal scar and one patient with retinal detachment. As many as $81.08 \%$ of patients were in the blind category at the preoperative examination. In the three-month follow-up, $23.80 \%$ of the patients were still included in the BCVA $<3 / 60$ category. Meanwhile, there was only one patient in the category $\mathrm{BCVA} \geq 6 / 12$ at the pre-operative examination, and it increased to $47.62 \%$ at the three-month follow-up examination. These results indicate that traumatic cataract extraction can improve visual acuity, but the risk of blindness can still occur.

In a study conducted in Ethiopia, Kinori et al. reported that only $10 \%$ of patients had final visual acuity $>20 / 40$. This could be due to the more complex ocular condition of the patients in the study and the longer duration between 
trauma and surgery compared to other studies [3]. Inadequate health facilities in developing countries and the lack of communication among children regarding trauma events and their visual condition are the causes of delays in treatment and affect the outcome of visual acuity [3] [6].

The limitation of this study is that the data were retrospective, resulting in unequal data and duration of follow-up. Examinations carried out by different examiner could also present a bias. Further prospective studies can be performed to assess visual acuity improvement, post-treatment complications, and prognosis in traumatic cataracts with longer follow-up duration. Further research regarding the biometric accuracy or residual refractive error after IOL implantation in pediatric traumatic cataract patients can also be carried out.

\section{Conclusion}

Traumatic cataracts diagnosed at the tertiary eye center in Indonesia mostly affect school-age boys performing activities outside buildings. There was an increased visual acuity after appropriate management, but there was still risk of blindness even after surgery. The causes and mechanisms of trauma must be evaluated in order to develop strategies to prevent the incidence of trauma in children. Children, parents, teachers and policyholders should be educated with essential information to reduce the incidence of trauma. Besides, it is necessary to carry out supervision or assistance during children's activities so that the incidence of pediatric eye trauma can be prevented, both inside and outside buildings.

\section{Conflict of Interest}

The authors declare no conflicts of interest regarding the publication of this paper.

\section{References}

[1] Cao, H., Li, L., Zhang, M. and Li, H. (2013) Epidemiology of Pediatric Ocular Trauma in the Chaoshan Region, China, 2001-2010. PLoS ONE, 8, e48377. https://doi.org/10.1371/journal.pone.0060844

[2] Khokhar, S., Gupta, S., Yogi, R., Gogia, V. and Agarwal, T. (2014) Epidemiology and Intermediate Term Outcomes of Open- and Closed-Globe Injuries in Traumatic Childhood Cataract. European Journal of Ophthalmology, 14, 124-130. https://doi.org/10.5301/ejo.5000342

[3] Kinori, M., Tomkins-Netzer, O., Wygnanski-Jaffe, T. and Ben-Zion, I. (2013) Traumatic Pediatric Cataract in Southern Ethiopia-Results of 49 Cases. Journal of American Association for Pediatric Ophthalmology and Strabismus, 17, 512-515. https://doi.org/10.1016/j.jaapos.2013.06.008

[4] Xu, Y.-N., Huang, Y.-S. and Xie, L.-X. (2013) Pediatric Traumatic Cataract and Surgery Outcomes in Eastern China: A Hospital-Based Study. International Journal of Ophthalmology, 6, 160-164.

[5] Gilbert, C. and Foster, A. (2001) Childhood Blindness in the Context of VISION 2020-The Right to Sight. Bulletin of World Health Organization, 79, 227-232. 
[6] Gogate, P., Shah, M., Kulkarni, A., Sahasrabudhe, M. and Patil, S. (2012) Causes, Epidemiology, and Long-Term Outcome of Traumatic Cataracts in Children in Rural India. Indian Journal of Ophthalmology, 60, 481-486. https://doi.org/10.4103/0301-4738.100557

[7] Adlina, A., Chong, Y. and Shatriah, I. (2014). Clinical Profile and Visual Outcome of Traumatic Paediatric Cataract in Suburban Malaysia: A Ten-Year Experience. Singapore Medical Journal, 55, 253-256. https://doi.org/10.11622/smedj.2014067

[8] Zimmerman, A., Magalhães, I.H., Tanaka, H.A., Zimmermann, I.T. and Arieta, C.E.L. (2019) Pediatric Traumatic Cataract Review: Origin of the Trauma. Revista Brasileira de Oftalmologia, 78, 103-106. https://doi.org/10.5935/0034-7280.20180105

[9] Reddy, A.K., Ray, R. and Yen, K.G. (2009) Surgical Intervention for Traumatic Cataracts in Children: Epidemiology, Complications, and Outcomes. Journal of American Association for Pediatric Ophthalmology and Strabismus, 13, 170-174. https://doi.org/10.1016/j.jaapos.2008.10.015

[10] Good, W.V. and Carden, S.M. (2017) Ch: Accidental Trauma in Children. In: Taylor and Hoyt's Pediatric Ophthalmology and Strabismus, Elsevier, Toronto, 715-724.

[11] Shah, M.A. (2015) Traumatic Cataracts in Children: Visual Outcome. World Journal of Ophthalmology, 5, 80-85. https://doi.org/10.5318/wjo.v5.i2.80

[12] Du, Y., He, W., Sun, X., Lu, Y. and Zhu, X. (2018) Traumatic Cataract in Children in Eastern China: Shanghai Pediatric Cataract Study. Scientific Reports, 8, Article No. 2588. https://doi.org/10.1038/s41598-018-20982-1

[13] Adhikari, S., Badhu, B., Bhatta, N., Jha, C., Baral, N. and Kumari, N. (2007) Etiology and Clinical Profile of Pediatric Cataract in a Tertiary Care Center in Eastern Nepal. Journal of the Nepal Medical Association, 46, 94-98.

[14] Staffieri, S.E., Ruddle, J.B. and Mackey, D.A. (2010) Rock, Paper and Scissors? Traumatic Paediatric Cataract in Victoria 1992-2006. Clinical \& Experimental Ophthalmology, 38, 237-241. https://doi.org/10.1111/j.1442-9071.2010.02236.x

[15] Shenoy, B.H., Mittal, V., Gupta, A., Sachdeva, V. and Kekunnaya, R. (2013) Refractive Outcomes and Prediction Error Following Secondary Intraocular Lens Implantation in Children: A Decade-Long Analysis. British Journal of Ophthalmology, 97, 1516-1519. https://doi.org/10.1136/bjophthalmol-2012-302775

[16] Shah, M.A., Shah, S.M., Shah, A.H. and Pandya, J.S. (2014) Visual Outcome of Cataract in Pediatric Age Group: Does Etiology Have a Role? European Journal Ophthalmology, 24, 76-83. https://doi.org/10.5301/ejo.5000309

[17] Shah, M.A., Shah, S.M., Appleware, A.H., Patel, K.D., Rehman, R.M. and Shikhange, K.A. (201) Visual Outcome of Traumatic Cataract in Pediatric Age Group. European Journal of Ophthalmology, 22, 956-963. https://doi.org/10.5301/ejo.5000111

[18] Sen, P., Shah, C., Sen, A., Jain, E. and Mohan, A. (2018) Primary versus Secondary Intraocular Lens Implantation in Traumatic Cataract after Open-Globe Injury in Pediatric Patients. Journal of Cataract \& Refractive Surgery, 44, 1446-1453. https://doi.org/10.1016/j.jcrs.2018.07.061

[19] Gradin, D. and Yorston, D. (2001) Intraocular Lens Implantation for Traumatic Cataract in Children in East Africa. Journal of Cataract \& Refractive Surgery, 27, 2017-2025. https://doi.org/10.1016/S0886-3350(01)00823-9

[20] Aldakaf, A., Almogahed, A., Bakir, H. and Carstocea, B. (2006) Intraocular Foreign Bodies Associated with Traumatic Cataract. Oftalmologia, 50, 90-94. 